\title{
SETS WHICH SEPARATE SPHERES
}

\author{
JOHN W. KEESEE
}

An extended version of the Jordan curve theorem [1] states that if $X$ and $Y$ are respectively an $n$-manifold and an $(n+1)$-sphere and $X$ is contained in $Y$, then $Y-X$ is the union of two disjoint, open, connected sets each having $X$ as point set boundary. It is shown here that it is possible to relax the requirement that $X$ be an $n$-manifold in such a way that the conclusions continue to hold. Actually, topological conditions on $X$ will be given that are necessary and suffcient for $X$ to separate the $(n+1)$-sphere $S^{n+1}$ in the stated manner.

A cohomology theory is assumed to be defined on the category of compact pairs and to satisfy the continuity property as well as the axioms of Eilenberg and Steenrod [2]. Spanier [3] has given one of several ways of showing the existence of such a theory having an arbitrary abelian coefficient group $G$ (discrete).

For such a cohomology theory an $n$-manifold $X$ contained in $S^{n+1}$ has the following well known properties which are assumed here without proof:

(1) $H^{n}(X)$ is isomorphic to $G$;

(2) $H^{n}(A)=0$ for every closed proper subset $A$ of $X$.

It will be shown that for any closed subset $X$ of $S^{n+1}$, (1) and (2) are necessary and sufficient conditions for $S^{n+1}-X$ to be the union of two disjoint, open, connected sets each having $X$ as point set boundary.

For $n=0, H^{n}(X)$ is taken to be the reduced zero-dimensional group.

The material in $\S 1$ is contained in the Tulane University lecture notes of A. D. Wallace.

1. If $(X, A)$ is a compact pair the inclusion map

$$
i: A \rightarrow X
$$

induces the homomorphism

$$
i^{*}: H^{n}(X) \rightarrow H^{n}(A) .
$$

An element $e$ in $H^{n}(A)$ is said to be extendable to the element $e^{\prime}$ in $H^{n}(X)$ if $e=i^{*}\left(e^{\prime}\right)$. If attention is not directed to the specific element $e^{\prime}$, the situation can be described by saying that $e$ can be extended to $X$. For an element $e^{\prime}$ in $H^{n}(X)$ the element $i^{*}\left(e^{\prime}\right)$ is de-

Received by the editors July 27, 1953. 
noted by $e^{\prime} \mid A$. The set $A$ is said to support $e^{\prime}$ if $e^{\prime} \mid A$ is not zero. If $e^{\prime}$ is not zero but $e^{\prime} \mid A$ is zero for every closed proper subset $A$ of $X$, then $X$ is said to be a minimal support for $e^{\prime}$.

For a cohomology theory satisfying the continuity property on the category of compact pairs, every compact triad $\left(X ; X_{1}, X_{2}\right)$ is a proper triad and the Mayer-Vietoris sequence [2, p. 43],

$$
\stackrel{\phi}{\rightarrow} H^{n}\left(X_{1}\right)+H^{n}\left(X_{2}\right) \stackrel{\psi}{\rightarrow} H^{n}\left(X_{1} \cap X_{2}\right) \stackrel{\Delta}{\rightarrow} H^{n+1}(X) \stackrel{\phi}{\rightarrow}
$$

is exact. It will be recalled that $X=X_{1} \cup X_{2}$ and that for an element $\left(e_{1}, e_{2}\right)$ in $H^{n}\left(X_{1}\right)+H^{n}\left(X_{2}\right)$,

$$
\psi\left(e_{1}, e_{2}\right)=e_{1}\left|X_{1} \cap X_{2}-e_{2}\right| X_{1} \cap X_{2} .
$$

Theorem 1.1 (The extension theorem). If $(X, A)$ is a compact pair and e an element of $H^{n}(A)$, then $e$ can be extended to $H^{n}(P)$ where $P$ is the closure of an open set containing $A$.

This is an easy consequence of the continuity property (compare $[4$, p. 247]).

Theorem 1.2. Let $X=X_{1} \cap X_{2}, Y=X_{1} \cup X_{2}$ where $Y$ is compact and $X_{1}$ and $X_{2}$ are closed in $Y$. If an element $e$ in $H^{n}(X)$ can be extended to $e_{1}$ in $H^{n}\left(X_{1}\right)$ and to $e_{2}$ in $H^{n}\left(X_{2}\right)$, then it can be extended to an element $e^{\prime}$ in $H^{n}(Y)$ so that $e^{\prime} \mid X_{1}=e_{1}$ and $e^{\prime} \mid X_{2}=e_{2}$.

Proof. Consider the Mayer-Vietoris sequence

$$
\stackrel{\Delta}{\rightarrow} H^{n}(Y) \stackrel{\phi}{\rightarrow} H^{n}\left(X_{1}\right)+H^{n}\left(X_{2}\right) \stackrel{\psi}{\rightarrow} H^{n}(X) \stackrel{\Delta}{\rightarrow}
$$

and let $e, e_{1}$ and $e_{2}$ be as in the statement of the theorem. Then $\psi\left(e_{1}, e_{2}\right)=e_{1}\left|X-e_{2}\right| X=e-e=0$ so that by exactness $\left(e_{1}, e_{2}\right)=\phi\left(e^{\prime}\right)$ for some $e^{\prime}$ in $H^{n}(Y)$. Now

$$
\begin{gathered}
e^{\prime} \mid X_{1}=e_{1}, \\
e^{\prime} \mid X_{2}=e_{2}, \\
e^{\prime}\left|X=\left(e^{\prime} \mid X_{1}\right)\right| X=e_{1} \mid X=e
\end{gathered}
$$

and the conclusions of the theorem are verified.

Definition. Let $X$ and $M$ be closed sets in the compact space $Y$ and let $e$ be an element of $H^{n}(X)$ which cannot be extended to $X \cup M$ but can be extended to $X \cup N$ for every closed proper subset $N$ of $M$. Then $M$ is called an irreducible membrane for e relative to $Y$.

Theorem 1.3. If $X$ is a closed subset of the compact space $Y$ and $e$ an element of $H^{n}(X)$ which cannot be extended to $Y$, then there is an 
irreducible membrane for e relative to $Y$.

Using the extension theorem the proof is as in $[5$, p. 32].

Theorem 1.4. If $(Y, X)$ is a compact pair and $M$ is an irreducible membrane for an element $e$ of $H^{n}(X)$ relative to $Y$ then:

(a) $\mathrm{Cl}(M-X)=M$;

(b) $X \cap M$ supports $e$.

Proof. (a) if $X \cap M$ contains a nonempty set $V$ open in $M$ let $N=M-V$. Then $e$ can be extended to $X \cup M=X \cup N$.

(b) If $e \mid X \cap M=0$, then this element can be extended to $e$ in $H^{n}(X)$ and to the zero element in $H^{n}(M)$ and then by 1.2 can be extended to $X \cup M$ in such a way as to provide an extension of $e$ to $X \cup M$.

Theorem 1.5. If $X$ is a closed subset of $S^{n+1}$ and $M$ is an irreducible membrane for an element e of $H^{n}(X)$, then $M-X$ is open.

Proof. Suppose $M-X$ is not open. Then there is a point $x$ of $M-X$ such that every neighborhood of $x$ intersects the complement of $M-X$. This point has a spherical neighborhood $U$ whose boundary $F(U)$ intersects the complement of $M-X$ and whose closure does not intersect $X$. Let $A=M \cap F(U), N_{1}=M-U, N_{2}=M \cap \bar{U}$. Then $N_{1}$ and $N_{2}$ are closed proper subsets of $M, N_{1} \cup N_{2}=M, N_{1} \cap N_{2}=A$. A then is a closed proper subset of the $n$-sphere $F(U)$. By hypothesis the element $e$ can be extended to an element $e^{\prime}$ in $H^{n}\left(X \cup N_{1}\right)$. Also $e^{\prime} \mid A=0$ since $H^{n}(A)=0$. Therefore $e^{\prime} \mid A$ can be extended to $e^{\prime}$ in $H^{n}\left(X \cup N_{1}\right)$ and to the zero element in $H^{n}\left(N_{2}\right)$ and then to $X \cup N_{1}$ $\cup N_{2}=X \cup M$ as in Theorem 1.2. But this extension would also be an extension of $e$ to $X \cup M$ which is impossible. Therefore $M-X$ is open.

2. Theorem 2.1. Let $M$ be an irreducible membrane for an element $e$ in $H^{n}(X)$. Then $M-X$ is connected.

Proof. Suppose that $M-X=P \cup Q$ where $P$ and $Q$ are non-empty separated sets. Then $\bar{P} \cap Q=\square$ implies that $\bar{P}-P$ is contained in $M \cap X$. Let $N_{1}=(M \cap X) \cup P, N_{2}=(M \cap X) \cup Q$. Then $\bar{N}_{1}$ $=(\mathrm{Cl}(M \cap X)) \cup \bar{P}=(M \cap X) \cup P \cup(\bar{P}-P)=(M \cap X) \cup P=N_{1}$. Likewise $\bar{N}_{2}=N_{2}$ so that $N_{1}$ and $N_{2}$ are closed proper subsets of $M$. Also $N_{1} \cup N_{2}=M, N_{1} \cap N_{2}=M \cap X$. Now $e$ can be extended to $X \cup N_{1}$ and to $X \cup N_{2}$ and hence by 1.2 to $X \cup M$ which is impossible. Therefore $M-X$ is connected.

THEOREM 2.2. If $X$ is a minimal support for an element e of $H^{n}(X)$ 
and $M$ is an irreducible membrane for this element such that $M-X$ is open, then $X$ is the boundary of $M-X$.

Proof. Let $A=M-X$ and $F(A)$ be the boundary of $A$. By 1.4, $\bar{A}=M$ and $M \cap X$ supports $e$. Since $X$ is a minimal support for $e$ it follows that $M \cap X=X$. Since $A$ is open, $F(A)=\bar{A}-A=M-(M-X)$ $=M \cap X=X$.

THEOREM 2.3. If $X$ is a closed subset of $S^{n+1}$ and is a minimal support for an element $e$ in $H^{n}(X)$, then there are irreducible membranes $M_{1}$ and $M_{2}$ for e relative to $S^{n+1}$ such that $M_{1}-X$ and $M_{2}-X$ are disjoint and $X=F\left(M_{1}-X\right)=F\left(M_{2}-X\right)$.

Proof. By 1.3 there is an irreducible membrane $M_{1}$ for $e$ in $S^{n+1}$ and according to $1.5,2.1$, and $2.2, M_{1}-X$ is open, connected, and $X=F\left(M_{1}-X\right)$. Now let $N_{1}=S^{n+1}-\left(M_{1}-X\right)$ and suppose that the element $e$ can be extended to $N_{1}$. Let $U$ be a spherical neighborhood whose closure is contained in $M_{1}-X$ and let $N_{2}=M_{1}-U$. Then $N_{1} \cup N_{2}=S^{n+1}-U=E^{n+1}$ where $E^{n+1}$ is an $(n+1)$-cell. The element $e$ can now be extended to $X \cup N_{2}$ since $N_{2}$ is a closed proper subset of $M_{1}$ and to $X \cup N_{1}$ by assumption. Since $X=N_{1} \cap N_{2}$ it follows that $e$ can be extended to $N_{1} \cup N_{2}=E^{n+1}$. But this is impossible since $H^{n}\left(E^{n+1}\right)=0$. Therefore $e$ cannot be extended to $N_{1}$ and there exists an irreducible membrane $M_{2}$ for $e$ relative to $N_{1}$ and hence relative to $S^{n+1}$. As in the case of $M_{1}, M_{2}-X$ is open, connected, and, $F\left(M_{2}-X\right)=X$. It is clear from the construction that $M_{1}-X$ and $M_{2}-X$ are disjoint.

REMARK. It will be noted that in the proof of 2.3 the assumption that $X$ was a minimal support for $e$ was used only to show that $X=F\left(M_{1}-X\right)=F\left(M_{2}-X\right)$. The existence of the sets $M_{1}$ and $M_{2}$ depended only on the presence of a nonzero element of $H^{n}(X)$. From this the necessary part of the next theorem follows easily.

THEOREM 2.4. If $X$ is a closed subset of $S^{n+1}$, then for $S^{n+1}-X$ to be connected it is necessary and sufficient that $H^{n}(X)=0$ (for any nontrivial coefficient group).

Proof of Sufficiency. Suppose that $S^{n+1}-X$ is not connected. Then $S^{n+1}-X=P \cup Q$ where $P$ and $Q$ are open, nonvacuous, and disjoint. Let $N_{1}=S^{n+1}-P, N_{2}=S^{n+1}-Q$. Then $N_{1} \cup N_{2}=S^{n+1}, N_{1}$ $\cap N_{2}=X$. Consider the following portion of the Mayer-Vietoris sequence:

$$
H^{n}(X) \stackrel{\Delta}{\rightarrow} H^{n+1}\left(S^{n+1}\right) \stackrel{\phi}{\rightarrow} H^{n+1}\left(N_{1}\right)+H^{n+1}\left(N_{2}\right)
$$


The group $H^{n+1}\left(N_{1}\right)+H^{n+1}\left(N_{2}\right)$ is zero since $N_{1}$ and $N_{2}$ are closed proper subsets of $S^{n+1}$. Then by exactness the homomorphism $\Delta$ maps $H^{n}(X)$ onto the nontrivial group $H^{n+1}\left(S^{n+1}\right)$ and $H^{n}(X)$ cannot be zero.

Theorem 2.5. Let $(X, A, B)$ be a compact triple with the boundary of $A$ contained in $B$. If for a fixed integer $p$, no nonzero element of $H^{p}(A)$ can be extended to $X$, then the homomorphism $i^{*}: H^{p}(A) \rightarrow H^{p}(B)$ induced by the inclusion map is an isomorphism into.

Proof. Let $e$ be a nonzero element of $H^{p}(A)$. Since $e$ cannot be extended to $X$ there is by 1.3 an irreducible membrane $M$ for $e$ relative to $X$. Then by $1.4, \mathrm{Cl}(M-A)=M$ so that $M \cap A=\mathrm{Cl}(M-A)$ $\cap A$. Now $M \cap A$ is contained in $B$ since it is contained in $F(A)$ $=\mathrm{Cl}(X-A) \cap A$. Also, by $1.4, e \mid M \cap A \neq 0$ and it follows that $e \mid B$ $\neq 0$. Therefore the kernel of $i *$ is zero.

The following notation is introduced to facilitate the statement of the next proposition. Let $X$ be a closed set in $S^{n+1}$ and denote by $Q$ a fixed component of $S^{n+1}-X$. The remaining components of $S^{n+1}-X$ form a countable set and are denoted by $P_{0}, P_{1}, P_{2}, \cdots$. For each set $P_{j}$ let $M_{j}=S^{n+1}-\left(Q \cup P_{j}\right)$ and let $I_{j}: X \subset M_{j}$ be the inclusion map. Denote by $\sum H^{n}\left(M_{j}\right)$ the weak sum of the groups $H^{n}\left(M_{j}\right)$. Then each element $u$ of $\sum H^{n}\left(M_{j}\right)$ has a coordinate in each $H^{n}\left(M_{j}\right)$ and all but a finite number of these coordinates are zero. Define the homomorphism

$$
I: \sum H^{n}\left(M_{j}\right) \rightarrow H^{n}(X)
$$

by $I(u)=\sum I_{j}^{*}\left(u_{j}\right)$ where $u_{j}$ is the coordinate of $u$ in $H^{n}\left(M_{j}\right)$.

THEOREM 2.6. The homomorphism $I: \sum H^{n}\left(M_{j}\right) \rightarrow H^{n}(X)$ is an isomorphism onto.

Proof. (a) Kernel of $I=0$. Let $u$ be an element of $\sum H^{n}\left(M_{j}\right)$ with coordinates $u_{j}$ and suppose that $I(u)=0$. Let $m$ be a positive integer large enough that the set $0,1,2, \cdots, m$ contains the indices of all nonzero coordinates of $u$. Then $I(u)=0=\sum_{j=0}^{m} I_{j}^{*}\left(u_{j}\right)$ implies that $I_{0}^{*}\left(u_{0}\right)=\sum_{j=1}^{m} I_{j}^{*}\left(-u_{j}\right)=e$. Now the element $e$ can be extended to $u_{0}$ in $H^{n}\left(M_{0}\right)$ and to $\sum_{j=1}^{m}\left[-u_{j} \mid\left(X \cup P_{0}\right)\right]$ in $H^{n}\left(X \cup P_{0}\right)$. Also $M_{0}$ $\cap\left(X \cup P_{0}\right)=X$ and $M_{0} \cup\left(X \cup P_{0}\right)=S^{n+1}-Q$ so that, by 1.2 , $e$ can be extended to $S^{n+1}-Q$. But by virtue of $2.4, H^{n}\left(S^{n+1}-Q\right)=0$ since $Q$ is connected. Therefore $e=I_{0}^{*}\left(u_{0}\right)=0$. By $2.5, I_{0}^{*}$ is an isomorphism into since $X$ contains the boundary of $M_{0}$. Therefore $u_{0}=0$ and a similar argument shows that $u_{j}=0$ for $j=1,2, \cdots, m$. Hence $u$ is the zero of $\sum H^{n}\left(M_{j}\right)$ and the kernel of $I=0$. 
(b) $I$ is onto. The proof will be made first for the case where the number of components $P_{j}$ is finite. If $P_{0}$ is the only such set, then $M_{0}=X$ and $I$ is the identity. Suppose inductively $I$ is onto when there are $k$ components $P_{j}$. Then if $Q, P_{0}, P_{1}, \cdots, P_{k}$ are the components of $S^{n+1}-X$, let $X_{0}=X \cup P_{0}$ so that $X_{0} \cup M_{0}=S^{n+1}-Q, X_{0}$ $\cap M_{0}=X$. The Mayer-Vietoris sequence of the triad $\left(X_{0} \cup M_{0}\right.$; $X_{0}, M_{0}$ ) contains the part

$$
H^{n}\left(X_{0}\right)+H^{n}\left(M_{0}\right) \stackrel{\psi}{\rightarrow} H^{n}(X) \stackrel{\Delta}{\rightarrow} H^{n+1}\left(S^{n+1}-Q\right) .
$$

Now $\psi$ maps $H^{n}\left(X_{0}\right)+H^{n}\left(M_{0}\right)$ onto $H^{n}(X)$ since $H^{n+1}\left(S^{n+1}-Q\right)$ is zero.

Next consider the following inclusion maps:

$$
\begin{aligned}
& I_{j}: X \subset M_{j} \quad(j=0,1, \cdots, k), \\
& I_{0, j}: X_{0} \subset M_{j} \quad(j=1,2, \cdots, k) \text {, } \\
& \alpha: X \subset X_{0} \text {. }
\end{aligned}
$$

Define the homomorphisms

$$
\begin{aligned}
& J: \sum_{j=0}^{k} H^{n}\left(M_{j}\right) \rightarrow H^{n}\left(X_{0}\right)+H^{n}\left(M_{0}\right), \\
& I_{0}: \sum_{j=1}^{k} H^{n}\left(M_{j}\right) \rightarrow H^{n}\left(X_{0}\right)
\end{aligned}
$$

by $J(u)=\left(\sum_{j=1}^{k} I_{0, j}^{*}\left(u_{j}\right),-u_{0}\right)$ and $I_{0}\left(u^{\prime}\right)=\sum_{j=1}^{k} I_{0, j}^{*}\left(u^{\prime}\right)$. First note that an element $u$ in $\sum_{j=0}^{\boldsymbol{k}} H^{n}\left(M_{j}\right)$ can be written uniquely as $u=\left(u_{0}, u^{\prime}\right), u_{0}$ in $H^{n}\left(M_{0}\right)$ and $u^{\prime}$ an element of $\sum_{j=1}^{k} H^{n}\left(M_{j}\right)$. Then $J(u)=J\left(u_{0}, u^{\prime}\right)=\left(I_{0}\left(u^{\prime}\right),-u\right)$. Now since $Q, P_{1}, \cdots, P_{k}$ are the components of $S^{n+1}-X_{0}, I_{0}$ is onto by the induction hypothesis. It is then clear that $J$ is also onto.

Next let $u$ be an element of $\sum_{j=0}^{k} H^{n}\left(M_{j}\right)$ in the following diagram:

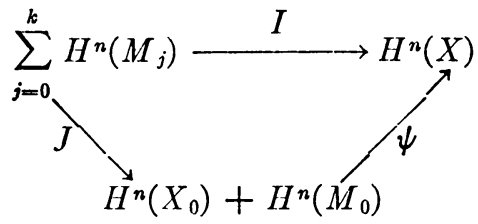

$I(u)=\sum_{j=0}^{\boldsymbol{k}} I_{j}^{*}\left(u_{j}\right)=\sum_{j=1}^{\boldsymbol{k}} I_{j}^{*}\left(u_{j}\right)+I_{0}^{*}\left(u_{0}\right)=\sum_{j=1}^{k} \alpha^{*} I_{0, j}^{*}\left(u_{j}\right)+I_{0}^{*}\left(u_{0}\right)$ $=\alpha^{*}\left[\sum_{j=1}^{k} I_{0, j}^{*}\left(u_{j}\right)\right]-\left[-I_{0}^{*}\left(u_{0}\right)\right]=\left[\sum_{j=1}^{k} I_{0, j}^{*}\left(u_{j}\right)\right]\left|X-\left[-u_{0}\right]\right| X$ $=\psi\left(\sum_{j=1}^{k} I_{0, j}^{*}\left(u_{j}\right),-u_{0}\right)=\psi J(u)$. Now since $\psi$ and $J$ are both onto and $I=\psi J$, it is seen that $I$ is onto when there are $k+1$ com- 
ponents $P_{j}$. This completes the proof for the finite case.

In the general case let $e$ be an element of $H^{n}(X)$. By 1.1, $e$ can be extended to a set $X_{1}$ where $X_{1}$ is the union of $X$ and all but a finite number of the sets $P_{j}$. Let $Q, P_{1}, P_{2}, \cdots, P_{k}$ be the components of $S^{n+1}-X_{1}$. In the following diagram $i^{*}$ is induced by the inclusion map, $I^{\prime}(u)=\sum_{j=0}^{k} I_{1, j}^{*}\left(u_{j}\right)$ where $I_{1, j}: M_{j} \subset X_{1}$ is the inclusion, and $\beta$ is defined by

$$
\begin{gathered}
\beta(u)_{j}= \begin{cases}u_{j} & \text { for } j \leqq k, \\
0 & \text { for } j>k .\end{cases} \\
\sum_{j=0}^{k} H^{n}\left(M_{j}\right) \stackrel{\beta}{\rightarrow} \sum H^{n}\left(M_{j}\right)
\end{gathered}
$$

By assumption there is an element $e^{\prime}$ of $H^{n}\left(X_{1}\right)$ such that $i^{*}\left(e^{\prime}\right)=e$ and since $I^{\prime}$ is onto, there is an element $u$ of $\sum_{j=0}^{k} H^{n}\left(M_{j}\right)$ for which $\boldsymbol{e}^{\prime}=I^{\prime}(u)$. Then $e=i^{*}\left(e^{\prime}\right)=i^{*} I^{\prime}(u)=i^{*}\left[\sum_{j=0}^{\boldsymbol{k}} I_{1, j}^{*}\left(u_{j}\right)\right]=\sum_{j=0}^{\boldsymbol{k}} i^{*} I_{1, j}^{*}\left(u_{j}\right)$ $=\sum_{j=0}^{k} I_{j}^{*}\left(u_{j}\right)=I \beta(u)$. This shows that $I$ is onto in the general case. Next let $\Lambda=\{\lambda\}$ be the set of indices of the components of $S^{n+1}-X$. Also denote by $\sum_{\lambda} G_{\lambda}$ the weak product of a collection of groups indexed by $\Lambda$ where each group $G_{\lambda}$ is isomorphic to the coefficient group $G$.

Theorem 2.7. $H^{n}(X)$ is isomorphic to $\sum_{\lambda} G_{\lambda}$.

Proof. As before let $M_{j}=S^{n+1}-\left(P_{j} \cup Q\right)$ and define $R_{j}=S^{n+1}-P_{j}$ and $T_{j}=S^{n+1}-Q$. Consider the Mayer-Vietoris sequence of the triad $\left(S^{n+1} ; R_{j}, T_{j}\right)$ :

$$
\stackrel{\phi}{\rightarrow} H^{n}\left(R_{j}\right)+H^{n}\left(T_{j}\right) \stackrel{\psi}{\rightarrow} H^{n}\left(M_{j}\right) \stackrel{\Delta}{\rightarrow} H^{n+1}\left(S^{n+1}\right) \stackrel{\phi}{\rightarrow} .
$$

By virtue of 2.4, $H^{n}\left(R_{j}\right)$ and $H^{n}\left(T_{j}\right)$ are zero since $P_{j}$ and $Q$ are connected. Also $H^{n+1}\left(R_{j}\right)$ and $H^{n+1}\left(T_{j}\right)$ are zero since $R_{j}$ and $T_{j}$ are closed proper subsets of $S^{n+1}$. By exactness then, $\Delta: H^{n}\left(M_{j}\right)$ $\approx H^{n+1}\left(S^{n+1}\right)$. Hence each $H^{n}\left(M_{j}\right)$ is isomorphic to $G$ and the conclusion follows from 2.6.

THEOREM 2.8. Let $X$ be a closed subset of $S^{n+1}$. Then necessary and sufficient conditions that $S^{n+1}-X=P \cup Q$ where $P$ and $Q$ are disjoint, open, connected, and $X=F(P)=F(Q)$ are:

(1) $H^{n}(X) \approx G$ for every coefficient group $G$,

(2) $H^{n}(A)=0$ for every closed proper subset $A$ of $X$. 
Proof. (a) Necessity. Condition (1) is a direct consequence of 2.7. As for (2) let $A$ be a closed proper subset of $X$ and set $R=P \cup(X-A)$, $T=Q \cup(X-A)$. Then $R$ and $T$ are respectively between the connected sets $P$ and $Q$ and their closures and hence are connected. Also $R \cup T=[P \cup(X-A)] \cup[Q \cup(X-A)]=P \cup Q \cup(X-A)$ $=(P \cup Q \cup X)-A=S^{n+1}-A, \quad R \cap T=[P \cup(X-A)] \cap[Q \cup(X-A)]$ $=X-A \neq \square$. Thus $S^{n+1}-A$, being the union of two intersecting connected sets $R$ and $T$, is connected and, by $2.4, H^{n}(A)=0$. This completes the proof of necessity.

(b) Sufficiency. Let the integers be used as coefficient group and then (1) implies that $S^{n+1}-X$ is the union of two components $P$ and $Q$ since otherwise, by $2.7, H^{n}(X)$ would not be isomorphic to the coefficient group.

Next choose a nonzero element $e$ of $H^{n}(X) . X$ then is a minimal support for $e$ by virtue of (2). Let $M_{1}$ and $M_{2}$ be irreducible membranes for $e$ as in 2.3. The set $M_{1}-X$, being connected, is contained in the component $P$, say, of $S^{n+1}-X$. Then since $P \cap M_{1}=P$ $\cap\left(M_{1}-X\right)=M_{1}-X$, it is seen that $M_{1}-X$ is open and closed in $P$. Therefore $M_{1}-X=P$ since $P$ is connected. Likewise $M_{2}-X=Q$ and it follows that $X=F(P)=F(Q)$.

REMARK. Theorem 2.6 is equivalent to a result of Eilenberg and Steenrod [2, Theorem 6.10, p. 319]. Also a theorem of Borsuk's [6, p. 240] is the same as 2.7 except that Borsuk's proposition involves cohomotopy groups. A comparison shows that for a closed subset of $S^{n+1}$, the $n$-dimensional cohomotopy and integral cohomology groups are isomorphic for $n$ greater than 1 .

\section{BiBLIOGRAPHY}

1. L. E. J. Brouwer, Beweis des Jordanschen Satzes für den n-dimensionalen Raum, Math. Ann. vol. 71 (1912) pp. 314-319.

3. E. H. Spanier, Cohomology theory for general spaces, Ann. of Math. vol. 49 (1948) pp. 407-427. 249.

4. J. W. Keesee, On the homotopy axiom, Ann. of Math. vol. 54 (1951) pp. 247-

5. A. D. Wallace, A theorem on end points, Anais da Acad. Brasil. de Ciencias vol. 22 (1950) pp. 29-33.

6. K. Borsuk, Set theoretical approach to the disconnection theory of the euclidean space, Fund. Math. vol. 37 (1950) pp. 217-241.

University of Arkansas 